\title{
Twenty-four-hour retention of chlordiazepoxide (Librium) -attenuated threat behavior in male Siamese fighting fish (Betta splendens)
}

\author{
MICHAEL H. FIGLER \\ Laboratory of Psychobiology, Department of Psychiatry, University of California \\ San Francisco, California 94143
}

\begin{abstract}
Experiment I investigated the 24-h retention of chlordiazepoxide (Librium) -induced attenuation and facilitation of habituation of species-specific threat behavior in male Siamese fighting fish. In Session 1, subjects, in a drug solution or plain water, were exposed to an undrugged conspecific stimulus fish for $40 \mathrm{~min}$. After $24 \mathrm{~h}$ back in their home jars, all fish were retested in plain water with the same stimulus fish. Chlordiazepoxide showed similar threat behavior attenuation in Sessions 1 and 2 as compared to the control situation. In Experiment II, subjects were immersed in one of the two treatments for the same time period with no stimulus fish present. After $24 \mathrm{~h}$ in their home environment, all fish were tested in plain water with a stimulus fish present. Chlordiazepoxide attenuated threat behavior and facilitated its habituation $24 \mathrm{~h}$ after initial exposure to the drug. Therefore, the results of Experiment I are due to sustained drug activity rather than transfer of drug-induced behavior from a drugged to a nondrugged state. Suggestions for further research, based upon this sustained drug activity, are discussed.
\end{abstract}

It has been demonstrated that chlordiazepoxide (Librium), a benzodiazepine class antianxiety agent, attenuates the agonistic (threat-attack) behavior of male Siamese fighting fish, a highly aggressive member of the family Belontiidae (Figler, 1973; Figler, Klein, \& Radford, 1973; Figler, Klein, \& Thompson, 1975). Figler (1973) found attenuated threat display behavior to a live, undrugged conspecific when subjects were immersed in a 15- or $30-\mu \mathrm{g} / \mathrm{ml}$ drug solution, doses which produced no measurable behavioral toxicity. Facilitation of habituation of the display was also reported, along with dose-response data for both the threat attenuation and facilitation of habituation effects. Further research showed that chlordiazepoxide (CDZ) also decreased actual attack behavior (biting, jawlocking) of conspecifics paired in direct encounters (Figler et al., 1973; Figler et al., 1975), using the same doses. Separate data showed no effects of these doses on respiration, locomotion, or feeding when subjects were isolated in drug solutions for equivalent time periods (Experiment II, Figler et al., 1975).

It has previously been shown that under nondrug conditions, using similar time parameters and the

The research was conducted while the author was a participant in the NSF Summer Post-doctoral Research Program for College Teachers at the University of Michigan, working with Dr. R. E. Davis. The manuscript was prepared while the author was a U.S.P.H.S. postdoctoral research fellow (No. MH-7082) in the Laboratory of Psychobiology, University of California-San Francisco, with Dr. H. V. S. Peeke. Reprints should be addressed to the author, who is now at the Department of Psychology, Towson State College, Baltimore, Maryland 21204. Portions of this research were presented at the meeting of the Eastern Psychological Association, Washington, D.C., May 1973. same eliciting stimulus fish class (live, responsive conspecific) as in the above drug studies, there is no retention of the effects of a 40-min threat display habituation session when fish are retested $24 \mathrm{~h}$ later with the same stimulus fish (Figler, 1972).

\section{EXPERIMENT I}

Using one $(30 \mu \mathrm{g} / \mathrm{ml})$ dose only, Experiment I was an attempt to ascertain whether the CDZ-induced attenuation of threat behavior is significantly retained for a 24-h interval as compared to a control situation (cf. Figler, 1972). Although no such data exist for fish, Randall (1961) reported that CDZ is present in the blood of some mammalian species (e.g., rats, dogs, humans) for several days after the original dose is given. Antiagonistic action of CDZ in mammals has also previously been demonstrated (see Figler, 1973).

\section{Method}

Subjects. Thirty adult male Siamese fighting fish, weighing approximately $2 \mathrm{~g}$ and measuring $4 \mathrm{~cm}$ in length (excluding fins), were individually isolated in 3.78-liter translucent plastic jars for 2 weeks prior to and throughout testing. Water temperature was held at $27^{\circ}-28^{\circ} \mathrm{C}$ by thermostatically regulating the laboratory air temperature. An automatically controlled timer provided $12 \mathrm{~h}$ of light and $12 \mathrm{~h}$ of darkness. All other details of housing and maintenance have been described elsewhere (Figler, 1972, 1973).

Apparatus. Subject testing was conducted in a transparent Plexiglas aquarium having outside measurements of $24.13 \times 12.70 \mathrm{x}$ $20.96 \mathrm{~cm}$. As previously illustrated (Figler, 1973), this .64-cm-thick Plexiglas aquarium was partitioned off by a clear, watertight Plexiglas sheet into two compartments with inside lengths of 19.69 and $2.54 \mathrm{~cm}$, respectively. The larger compartment held the subject, with the smaller compartment enclosing the live conspecific male stimulus fish. The test aquarium was placed within a viewing amphitheater similar to the one described by Figler (1973). 


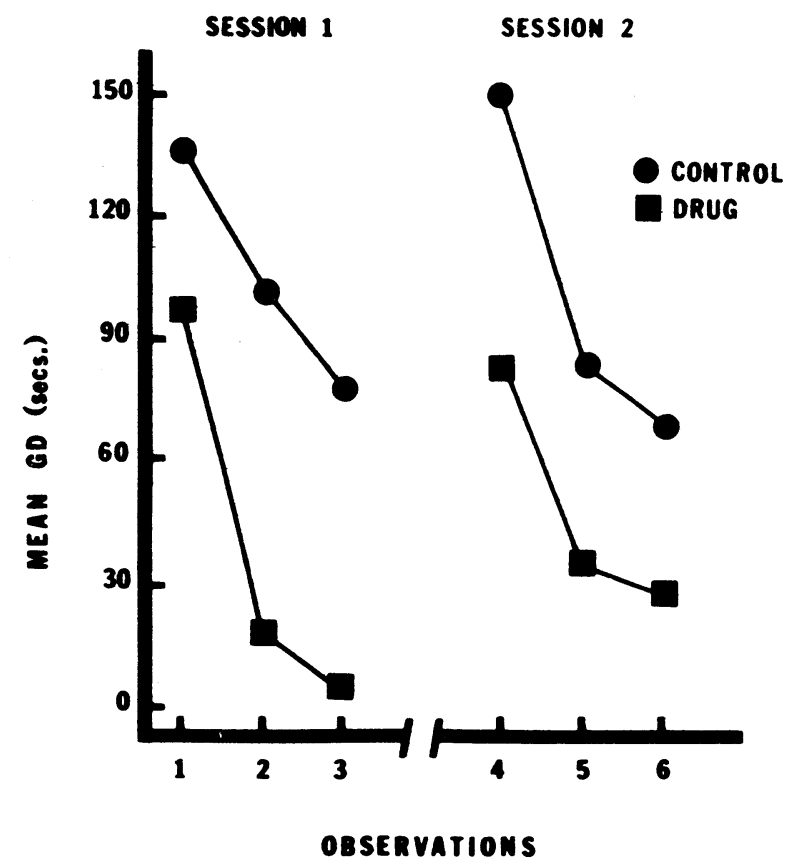

Figure 1. Mean gill-cover erection duration (seconds) per 10-min observation period.

Behaviors were recorded on an Esterline-Angus event recorder, two channels being exclusively activated by individual on-off push buttons embedded in a wooden box. A Graylab Universal timer automatically controlled the duration and termination of the experimental sessions.

Procedure. The fish were randomly assigned to one of three independent groups $(\mathrm{N}=10)$ : (1) $30 \mu \mathrm{g} / \mathrm{ml}$ group, (2) tap water control group, and (3) eliciting stimulus group.

Each subject was tested in the subject compartment in 3 liters of aged tap water containing either $30 \mu \mathrm{g} / \mathrm{ml}$ of dissolved chlordiazepoxide or no drug at all (control). The stimulus fish compartment was filled to the same level with aged tap water in both conditions. Therefore, eliciting stimulus fish were never exposed to the drug. Each stimulus fish was randomly assigned to two different subject fish during the experiment.

As in Figler (1973), each subject was placed in the prefilled test aquarium which an assistant had prepared beforehand. The experiment was therefore a "blind" one, since chlordiazepoxide is colorless and water soluble. The fish was acclimated to the surroundings for $30 \mathrm{~min}$, being separated from the stimulus fish by a removable opaque partition behind the permanent watertight Plexiglas partition. The opaque partition was then removed, and a 40-min test session was initiated. After testing, both fish were returned to their home jars for a 24 -h period. Then Session 2 was conducted using the same stimulus fish as that used in Session 1. The procedures of the two sessions were identical except that all subjects were tested during Session 2 in aged tap water containing no drug.

During each of the 40-min sessions, three 10 -min event recordings (separated by $5 \mathrm{~min}$ ) of the subject's threat behavior were taken. Two widely used and highly reliable components of the threat display and an arousal measure were simultaneously recorded (Adler \& Hogan, 1963; Clayton \& Hinde, 1968; Figler, 1972, 1973; Shapiro \& Schuckman, 1971; Simpson, 1968). For a detailed description of $B$. splendens' agonistic sequence, see Figler (1973). (1) Gill-cover erection duration (GD): the total amount of time that the gill covers were erected during the 10 -min period. (2) Gill-cover erection frequency (GF): the number of times the gill covers were erected during the $10-\mathrm{min}$ period. (3) Airgulping frequency $(\mathrm{AG})$ : the number of times the fish came to the surface to gulp air during the $10-\mathrm{min}$ period. This behavior is not a direct component of the threat display, itself, but is an arousal level measure (see Figler, 1972, 1973, for a more thorough discussion of this behavior).

\section{Results}

A three-factor ( 2 treatments by 3 trials by 2 sessions) mixed-design analysis of variance was performed for each dependent variable (Winer, 1971).

The treatment effect for the gill-cover erection duration data was highly significant $[F(1,18)=$ $14.83, \mathrm{p}<.01]$, with the drug group having an overall lower level of responding for sessions combined (see Figure 1). A highly significant trials effect reflected an across-observations decrement for the combined treatment groups $[\mathrm{F}(2,36)=26.95, \mathrm{p}<.01]$. A nonsignificant Treatment by Sessions interaction $[\mathrm{F}(1,18)=1.92, \mathrm{p}>.05]$ reflected the similarity of the treatment effect in Sessions 1 and 2. A significant Treatment by Sessions by Trials interaction $[F(2,36)$ $=4.70, \mathrm{p}<.05]$ reflected a greater difference in absolute response decrement between treatment groups in Session 1 as compared to Session 2.

The gill-cover erection frequency (GF) data showed trends almost identical to the GD data, an expected finding, since GD and GF are highly positively correlated measures of the threat display (Figler, 1972). A highly significant treatment effect $[F(1,18=$ $36.35, \mathrm{p}<.01]$, trials effect $[\mathrm{F}(2,36)=9.83$, $\mathrm{p}<.01]$, and Treatment by Sessions by Trials interaction $[F(2,36)=10.91, p<.01]$ were found. Also, similar to the GD data, the GF Treatment by Sessions interaction was nonsignificant $[F(1,18)=$ 1.29].

The highly significant airgulping frequency $(\mathrm{AG})$ treatment effect $[F(1,18)=62.59, p<.01]$ was due to the lessened overall AG (arousal) level for the drug group. However, the only other significant finding was a trials effect $[\mathrm{F}(2,36)=6.58, \mathrm{p}<.01]$, demonstrating an overall habituation trend (response decrement) for groups and sessions combined.

In a separate analysis, a proportion of GD decrement was calculated for each subject's data from each session, in order to give a relative measure of within-session decrement. The same formula was used for each session's data: (First 10-min observation Third 10-min observation)/(First 10-min observation).

Mann-Whitney $U$ tests were used to determine treatment differences and Wilcoxon matched-pair signed-ranks or sign tests (Siegel, 1956) were used to evaluate differences within each group. All significance levels reported are two-tailed.

There was a significantly greater proportion of GD decrement in Session 1 for the drug group as compared to the control group $(U=15, \mathrm{p}<.05)$. Proportional decrement data could not be compared for Session 2, since four of the drug group fish never 
displayed in Session 2. However, the two groups did not significantly differ $(U=27, p>.05)$ in the level of GD during the first observation of Session 2, while the drug group reached a significantly lower level of terminal responding (third observation) in that session $(U=15.50, \mathrm{p}<.02)$.

With respect to within-group comparisons, the control group showed no significant differences in proportional decrement in Session 1 as compared to Session 2 ( $p>.05 ;$ sign test), nor were there differences in terminal responding in Session 1 as compared to Session $2(\mathrm{~T}=15, \mathrm{p}>.05)$. There was no significant difference in GD level between the first 10-min observations in each session for the drug group ( $T=22, \mathrm{p}>.05)$.

In general, 7 of the 10 drug group subjects reached a zero level of threat responding during Session 1 . Four of these subjects never responded in Session 2. All control group subjects showed some level of threat displaying throughout both sessions. Since the GF data were so similar to the GD data, the above analyses were not performed for the GF measure.

Using the same formula for obtaining the proportion of decrement, it was found that the drug group had a significantly greater proportion of $A G$ decrement in Session 1, as compared to the control group $(U=18, p<.02)$. However, no significant difference in proportional decrement was found in Session $2(U=39.50, p>.05)$. Within-group proportional decrement analyses showed no significant differences in the Session 1-Session 2 comparison for either treatment group.

\section{EXPERIMENT II}

Although Experiment I showed significant 24-h retention of the Session 1 experience, it is not clear whether the Session 2 results were due to the continued behavioral activity of $\mathrm{CDZ}$ or a transfer of effects from a drug to nondrug state. Therefore, Experiment II was undertaken to determine if this dose of CDZ is behaviorally active $24 \mathrm{~h}$ after an isolated fish has been exposed to the drug for a 70-min period (the same drug exposure period as in Experiment I). In other words, can the CDZ-induced attenuation of threat behavior and facilitation of habituation occur $24 \mathrm{~h}$ after exposure to the drug?

\section{Method}

Subjects. Twenty-one experimentally naive adult male $B$. splendens were obtained through the same commercial source as Experiment I fish, and were housed and maintained in the same manner.

Apparatus. The test aquarium and viewing amphitheater were the same as those used in Experiment I. Also, the same recording and timing devices were used in the present experiment.

Procedure. The fish were assigned randomly to three independent groups $(\mathrm{N}=7)$ : (1) $30 \mu \mathrm{g} / \mathrm{ml}$ group, (2) tap water control group, and (3) eliciting stimulus group. In Session 1, the fish were put into the test aquarium containing 3 liters of aged tap water or 3 liters of a $30 \mu \mathrm{g} / \mathrm{ml} \mathrm{CDZ}$ solution, as in Experiment I. The stimulus compartment was filled to the same level with aged tap water in both treatment conditions. Unlike Experiment I, there was no stimulus fish used for either treatment group in Session 1. However, the subjects were put into the large compartment for $30 \mathrm{~min}$, with the opaque partition inserted, with that partition then being removed for an additional 40-min exposure period (as in Experiment I). After Session 1, the fish were returned to their home jars for $24 \mathrm{~h}$. Session 2 then took place with all subjects tested in tap water containing no drug, with an eliciting stimulus fish in its proper compartment. Therefore, the Session 2 procedure for Experiment II was similar to the Session 2 procedure in Experiment I. As in Experiment I, each stimulus fish was used twice during the present experiment.

During Session 2, the same three dependent measures (GD, GF, AG) were recorded during three 10-min observation periods, as in Experiment I. No behavioral measures were taken during Session 1, since in vacuo threat behavior rarely occurs (Figler, 1972) and since it has been previously shown that $30 \mu \mathrm{g} / \mathrm{ml}$ of CDZ produces no changes in airgulping, locomotion, or eating behavior in an identical isolation procedure (Figler et al., 1975).

\section{Results}

A two-factor ( 2 treatments by 3 observations) mixed-design analysis of variance was performed for each dependent variable.

The treatment effect for gill-cover erection duration (GD) was nonsignificant $[\mathrm{F}(1,6)=3.82, \mathrm{p}>.05]$, due to characteristic long-duration gill-cover erections by the drug group during the first 10-min observation period. This phenomenon has been previously reported for this dose (Figler, 1973). The highly significant trials effect $[\mathrm{F}(2,12)=19.31, \mathrm{p}<.01]$ showed the across-session response decrement. The Treatment by Trials interaction failed to reach significance $[\mathrm{F}(2,12)=3.58, \mathrm{p}>.05]$.

There was a highly significant gill-cover erection (GF) treatment effect $[F(1,6)=19.11, p<.01]$, with the drug group showing less overall responding (see Figure 2). There was also a significant across-session response decrement $[\mathrm{F}(2,12)=4.54, \mathrm{p}<.05]$, although no significant Treatment by Trials interaction was found $[\mathrm{F}(2,12)=2.92, \mathrm{p}>.05]$.

The airgulping frequency (AG) treatment effect $[F(1,6)=15.32, p<.01]$ reflected lessened overall airgulping in the drug group. However, the trials effect $[\mathrm{F}(2,12)=.65]$ and Treatment by Trials interaction $[F(2,12)=.70]$ did not reach significance.

Using the previously described formula (see Experiment I), a proportion of across-session decrement was calculated for the GD and GF data. A Mann-Whitney $\mathrm{U}$ test showed that the drug group had a significantly larger proportion of decrement than the control group $(U=5, p<.02)$. A similar finding was uncovered for the GF data $(U=7$, $\mathrm{p}<.03)$.

\section{DISCUSSION}

Chlordiazepoxide (CDZ) -induced attenuation of threat behavior in $B$. splendens is significantly retained for at least $24 \mathrm{~h}$. The effect 


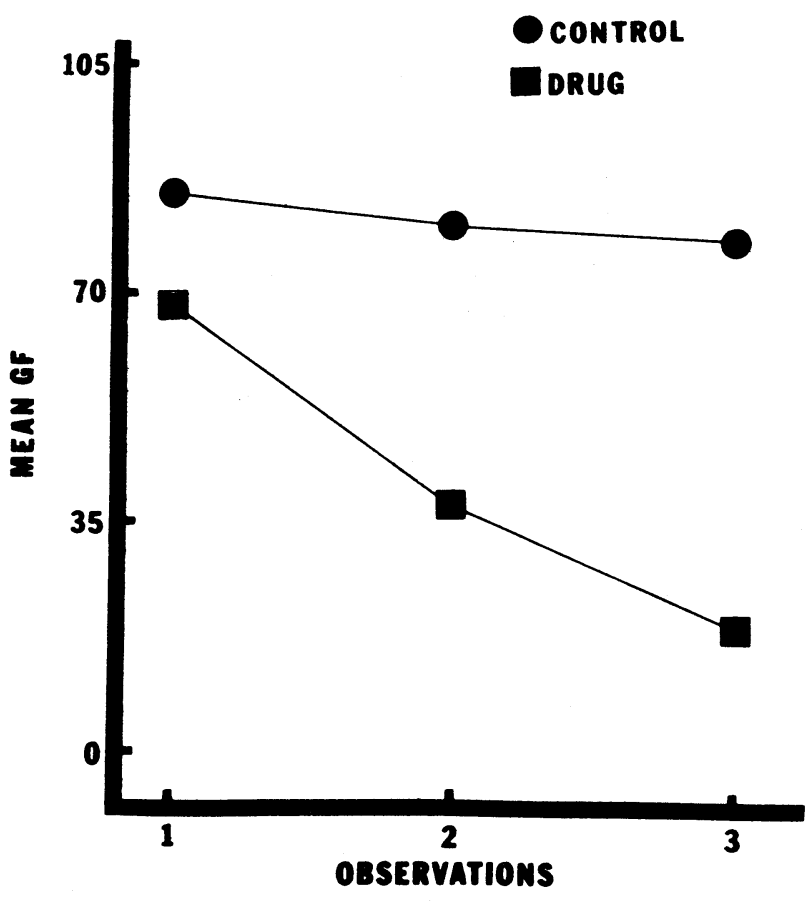

Figure 2. Mean gill-cover erection frequency per $10-\mathrm{mm}$ observation period.

is due to the continued behavioral activity of the drug, as shown in Experiment II, where CDZ also facilitated threat habituation $24 \mathrm{~h}$ after initial drug exposure. Although there is a dearth of studies on long-term retention of CDZ-induced modifications of behavior (e.g.. Oishi, Iwahara, Yang, \& Yogi, 1972), it is known that the drug remains present in the blood of mammals for long time periods (Randall, 1961). The present studies are the first known attempts to investigate retention of $\mathrm{CDZ}$ effects in fish. The long-term activity of $\mathrm{CDZ}$ on $B$. splendens' species-specific threat behavior remains to be demonstrated in other fish species. This sustained drug activity could be taken advantage of in acute investigations where the actual presence of the drug in the test environment may interfere with certain processes such as egg hatching (e.g., when drug effects on parental behaviors are being observed), or if only one fish in a paired encounter (e.g., mating) is to have had exposure to the drug.
By immersing the fish in the drug shortly before the experiment (e.g.. in a separate environment), these potentially confounding drug effects may be avoided. However, further research should determine the rate of absorption, site(s) of action, and time course of excretion of the drug in order to more precisely determine dose levels over time.

\section{REFERENCES}

Adler, N., \& Hogan, J. A. Classical conditioning and punishment of an instinctive response in Betta splendens. Animal Behaviour. $1963,11,351-354$.

Clayton, F. L., \& Hinde, R. A. The habituation and recovery of aggressive display in Betta splendens. Behaviour, 1968, 30, 96-106.

Figler, M. H. The relation between eliciting stimulus strength and habituation of the threat display in male Siamese fighting fish, Betta splendens. Behaviour, 1972, 42, 63-96.

Figler, M. H. The effects of chlordiazepoxide (Librium) on the intensity and habituation of agonistic behavior in male Siamese fighting fish. Psychopharmacologia, 1973, 33, 277-292.

Figler, M. H., Klein, R. M., \& Radford, R. B. The effects of chlordiazepoxide (Librium) on the attack behavior of male Siamese fighting fish, Betta splendens. Proceedings of the 81st Annual Convention of the American Psychological Association, 1973, 8, 1027-1028.

Figler, M. H., Klein, R. M., \& Thompson, C. S. Chlordiazepoxide (Librium)-induced changes in intraspecific attack and selected non-agonistic behaviors in male Siamese tighting fish. Psychopharmacologia, 1975, 42, 139-145.

Oishi, H., Iwahara, S., Yang, K., \& Yogi, A. Effects of chlordiazepoxide on passive avoidance responses in rats. Psychopharmacologia, 1972, 23, 373-385.

Randall, L. O. Pharmacology of chlordiazepoxide (Librium). Diseases of the Nervous System, 1961, 22, 7-15.

Shapiro, S., \& Schuckman, H. Habituation and covariation of the components of the threat display in Betta splendens. Psychological Reports, 1971, 28, 827-837.

SIEGEL, S. Nonparametric statistics for the behavioral sciences. New York: McGraw-Hill, 1956.

Simpson, M. J. A. The display of the Siamese fighting fish Betta splendens. Animal Behaviour Monographs, 1968, 1, (whole issue).

WINER, B. J. Statistical principles in experimental design (2nd ed.). New York: McGraw-Hill, 1971.

(Received for publication June 19, 1975.) 